\title{
Fully Convolutional Network based Ship Plate Recognition
}

\author{
Weishan Zhang \\ Department of Software Engineering \\ China University of Petroleum \\ Qingdao, China \\ zhangws@upc.edu.cn
}

Xin Liu

School Computer and Communication Engineering

China University of Petroleum

Qingdao, China

1x@upc.edu.cn

\author{
Haoyun Sun \\ Department of Software Engineering \\ China University of Petroleum \\ Qingdao, China \\ hys_upc@163.com
}

\author{
Jiehan Zhou \\ University of Oulu \\ Oulu, Finland \\ jiehan.zhou@oulu.fi
}

\author{
Zhanmin Zhang \\ Engineering Technology Research Institute \\ Huabei Oilfield Company, PetroChina \\ Renqiu, China \\ cyy_zhangzm@petrochina.com.cn
}

\author{
Guizhi Min \\ Engineering Technology Research Institute \\ Huabei Oilfield Company, PetroChina \\ Renqiu, China \\ cyy_mgz@petrochina.com.cn
}

\begin{abstract}
Ship plate recognition is challenging due to variations of plate locations and text types. This paper proposes an effcient Fully Convolutional Network based Plate Recognition approach FCNPR, which uses a CNN (Convolutional Neural Network) to locate ships, then detects plate text lines with the fully convolutional network (FCN). The recognition accuracy is improved with integrating the AIS (Automatic Identification System) information. The actual FCNPR deployment demonstrates that it can work reliably with a high accuracy for satisfying practical usages.

Index Terms-Ship license plate recognition, Text detection,
\end{abstract} Fully Convolutional Network, AIS

\section{INTRODUCTION}

Automatic ship monitoring plays an important role for smart port [1]. The emerging deep learning technologies [2] and increasing surveillance cameras provide a good chance for ship license plate recognition. There is no an effective method for Chinese plate recognition up to now. We propose a method named FCNPR to locate ships, and detect text lines with the fully convolutional network (FCN) [3], finally, recognize plates by text line classification. To improve recognition accuracy, information from AIS [4] (an automatic navigation system connecting base stations and ship borne equipment) is integrated to enrich the knowledge base for text line classification.

The contributions of this paper are:

- We propose a hybrid method called FCNPR that combines text detection and text line classification for ship license plate recognition to address challenges of complex background and object occlusion, especially variations of ship license plate locations and text types.
- We build an online adaptive architecture to enrich the knowledge base of text line classification for high recognition accuracy.

- We propose a method called FCNTLD (FCN based Text Line Detector) for text line detection. It directly predicts text lines in full images based on $\mathrm{FCN}$, and works efficiently and effectively for Chinese character detection.

- We have extensively evaluate FCNPR for its scalability, performance, accuracy and fault-tolerance.

The remainder of the paper is organized as follows: Section II discusses the related work. Section III presents the architectural design and details the implementation of FCNPR. Section IV discusses comprehensive evaluations of the deployed FCNPR solution. Section V gives the conclusion and the future work to be done.

\section{RELATED WORK}

\section{A. Character Recognition}

License plate recognition is one of the most typical application for smart world. Gou et al. [5] proposed a vehicle license plate recognition method based on character-specific extremal regions (ERs) and hybrid discriminative restricted Boltzmann machines (HDRBMs). They used vertical edge detection tophat transformation, morphological operations, and various validations to detect license plates to extract specific characters with ERs from detection results, and recognize the characters by the offline trained pattern classifier of HDRBM.

To solve the problem of distortion on ship plates introduced by camera location, Hui et al. [6] presented a novel contour 
tracking method to remove the redundant information, and then used the Hough transform to detect straight lines to speed up the processing speed. They determined the border of the license plate image with a lint fitting algorithm, which could obtain 4 vertices of the license plate region. Finally, they corrected images by bilinear space changes. Their focus is different from ours. We use text line classification for license plate recognition, while their work focused on regulate characteristics angles to improve recognition accuracy.

Lin et al. [7] proposed a method to learn strokes, radicals and character features of Chinese characters based on Convolution Neural Network (CNN) to recognize Chinese Character CAPTCHAs (Completely Automated Public Turing test to tell Computers and Humans Apart), which performed better than Chinese Optical Character Recognition (OCR) [8]. It achieved over $95 \%$ accuracy for single Chinese character and $84 \%$ accuracy for three types of Chinese Character CAPTCHAs with four Chinese characters. However, this method can only work for printed characteristics.

\section{B. Deep learning}

Text detection is the key step for ship license plate recognition. The detection accuracy significantly affects the performance of the whole system. EAST (An Efficient and Accurate Scene Text Detector) is a simple and powerful pipeline that yields fast and accurate text detection at natural scenes [9]. It directly predicts words or text lines of arbitrary orientations and quadrilateral shapes in full images, eliminates unnecessary intermediate steps with the fully convolutional network, and achieves the F-score of 0.7820 at $13.2 \mathrm{fps}$ with a 720 p resolution. However, EAST focuses on words detection rather than text lines, and at the same time, its multi-orientation detection can cause high false detection rates.

Comparing with convolutional neural networks, a fully convolutional network can have any input size. FCN adopts Deconvnet to up-sampling for last feature map, and restores the feature map to the same size of the input image, so that each pixel can be predicted. FCN shows better performance than $\mathrm{CNN}$ while it may cost more computing resources and time. Wang et al. proposed [10] a handwritten string recognition method based on FCN and applied it into different applications. However, the performance of the method cannot satisfy real-time image processing.

\section{FCNPR FOR SHIP PLATE RECOGNITION}

\section{A. Overview}

The work flow of the FCNPR is shown in Fig. 1. First, the FCNPR collects and decodes video streams from cameras, and then it locates ships by ship detection using a SSD (Single Shot MultiBox Detector) network [11]. The most important tasks are text detection and text recognition. The text detection detects text on ship plates from image frames in video streams using an adapted fully convolutional neural network.

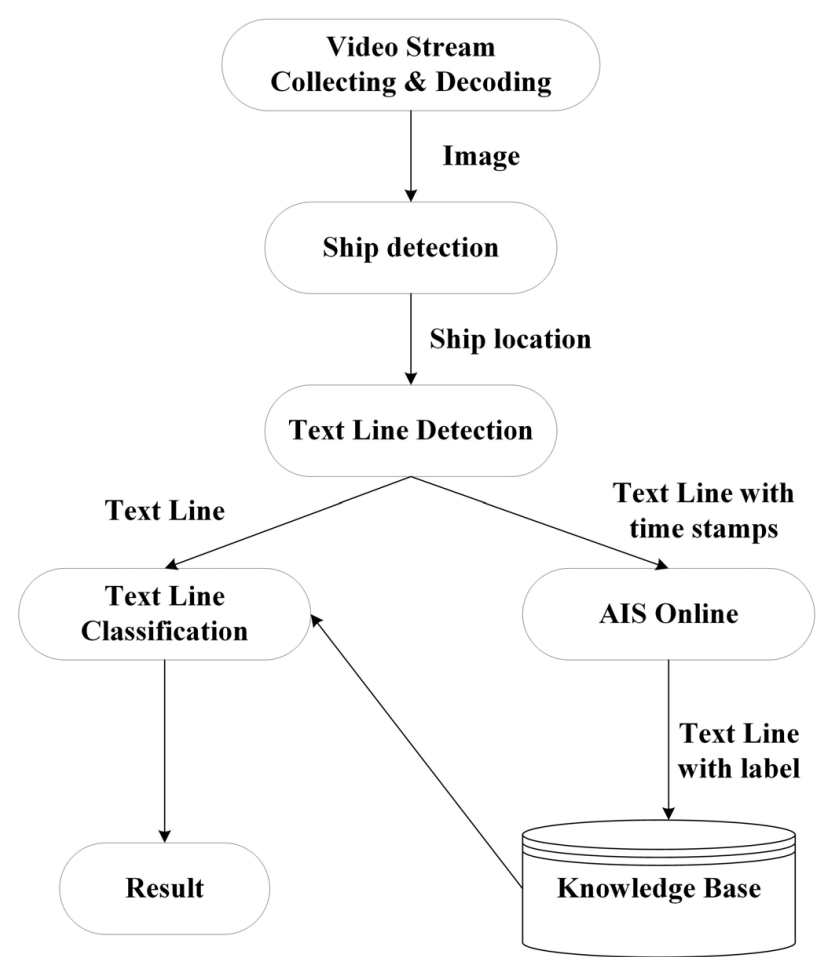

Figure 1: The FCNPR work flow

\section{B. Ship Detection}

FCNPR uses a kind of DCNN (Deep Convolutional Neural Network) called SSD for ship detection, which is a typical method of object detection including object localization and classification. SSD is good for target category prediction and bounding box localization. The implementation of SSD uses a small convolutional filter to predict object categories and offsets in bounding box locations, separates predictors (filters) for different aspect ratio detections.

The detection process is composed of offline and online processing as follows.

- Offline processing. Label the decoded images from video streams, and place labeled images into the knowledge base. And then FCNPR trains the detection model SSD using the labeled images in the knowledge base.

- Online processing. Locate ships in the images collected from on-site cameras.

\section{Text Line Detection}

EAST is good for text detection, which performs stateof-the-art in ICDAR 2015 and COCO-Text [9], its pipeline is shown in Fig. 2(A). However, comparing with English alphabet, Chinese characters and phrases are more complex. Due to variations of text types, EAST can not satisfy the requirement for Chinese text detection.

Inspired by the EAST and the FCN, we propose an improved text line detector, which is called FCNTLD (FCN based Horizontal Text Line Detector), as shown in Fig. 2 (B). 


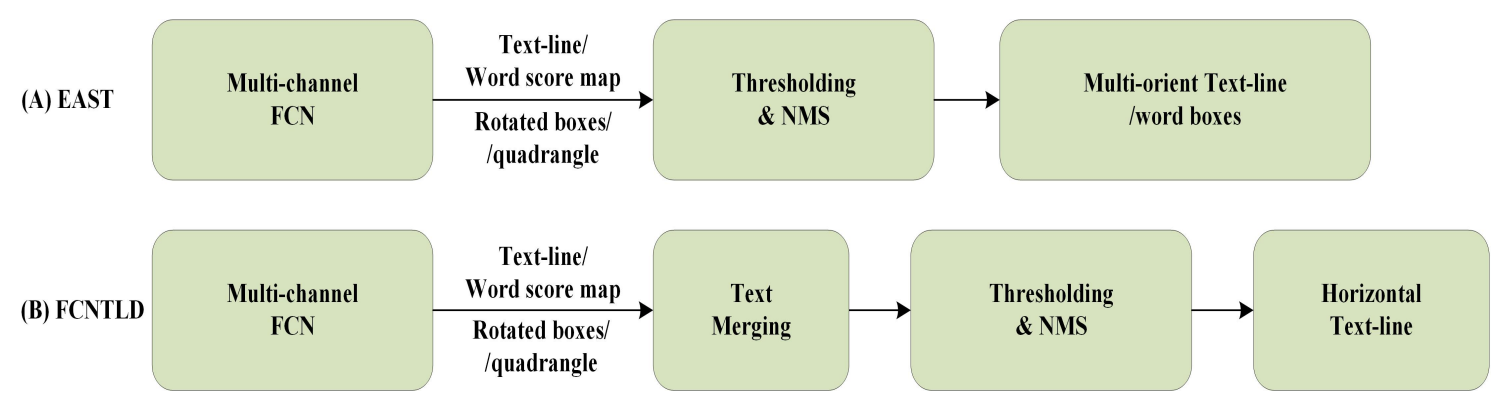

Figure 2: The architectures of EAST and FCNTLD

The FCNTLD has the following features::

- FCNTLD focuses on horizontal text detection.

- Algorithm merges the nearest pair of texts until there is no adjacent text. We calculate the distance between the center points of two text boxes as,

$$
X_{d}=\left(X_{r}^{2}-X_{l}^{2}\right) / w^{2}, Y_{d}=\left(Y_{r}^{2}-Y_{l}^{2}\right) / v^{2},
$$

where $X_{r}$ and $X_{l}$ are the x coordinate of text rectangle centers. $Y_{r}$ and $Y_{l}$ are the y coordinate of text rectangle centers, $w$ and $v$ are the weight of offset squares. We connect a sequence of detected text into a text line when $X_{d}<0.7$ and $Y_{d}<0.3$.

The text line detection is composed of offline training and online processing as follows.

- Offline training. Label a text with a quadrilateral on a plate, denote the four corner vertices of the quadrangle location with a $8 \mathrm{x} 1$ matrix $([\mathrm{x} 1, \mathrm{y} 1, \mathrm{x} 2, \mathrm{y} 2, \mathrm{x} 3, \mathrm{y} 3, \mathrm{x} 4, \mathrm{y} 4])$. The FCNTLD is trained end-to-end by the Tensorflow using the labeled images, the learning rate starts from 1e3 , decays to one-tenth every 12500 minibatches, and stops at $1 \mathrm{e}-6$. The FCNTLD is trained until its performance stops improving.

- Online processing. Detect text lines on plates collected from ship detection.

We have extensively evaluated the EAST and FCNPR for text line detection on ship as shown in Fig. 4. We test them with 1700 ship images at the same scene, the FCNTLD achieves 0.9630 accuracy and the EAST achieves 0.8922 accuracy, which proves that the FCNTLD performs better than the EAST on Chinese text line detection.

\section{Text Line Classification}

Chinese character recognition [12] has been an open study for several decades. So far, we have not found a general Chinese character recognition method for dealing with our case. Considering the limited number of ships in a port, we propose a method for text line classification, which is composed of offline training, online processing, and online adaptation as shown in Fig. 3.

- Offline training. Train classification models with data in the knowledge base using the CNN.

- Online processing. Categorize text lines with the trained classification model.

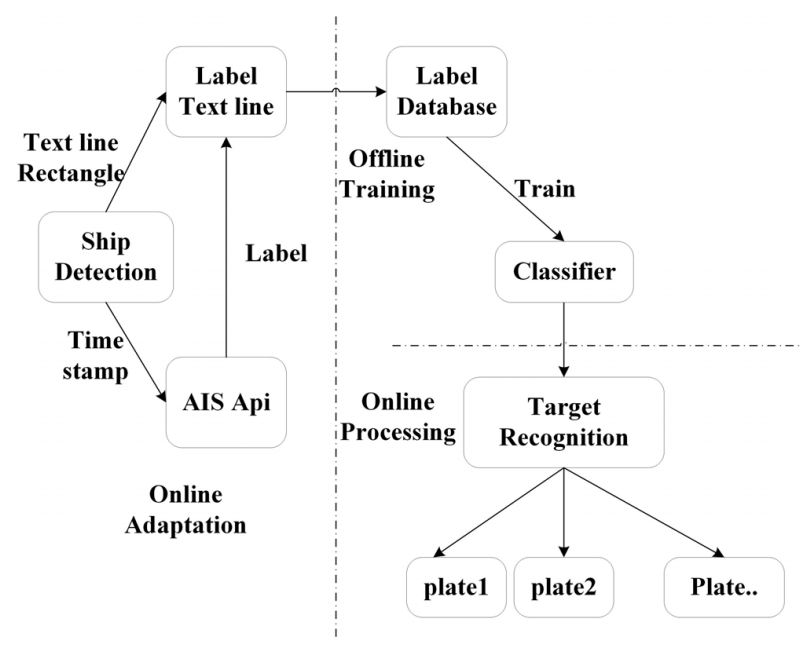

Figure 3: structure of text recognition

\section{E. Online Adaptation}

In FCNPR, this online adaptation updates the text line classifier online. It can make the classification algorithm more robust against appearance changes or plate deformations. The main components (as shown in Fig. 3) include:

- Collecting text line rectangles. We get the text lines locations in the Ship Detection step, then cut out the text lines.

- Labeling text line rectangles. Adding labels to text line rectangles, the labels are collected from AIS according to time stamps, which are the time points when text lines are detected.

- Update the text line classifier. Retraining and updating the text line classifier at night at preset intervals.

\section{$F$. The architecture of implementing the FCNPR}

We have built an adaptive real-time video processing system for ship plate recognition, with the FCNPR architecture shown in Fig. 5. In Offline Training, we label images into the knowledge base, train ship detection models with the SSD, train text detection model with the FCNTLD, and train the text line classifier with the CNN. Online Processing consists of two layers. The top layer collects video data through video 


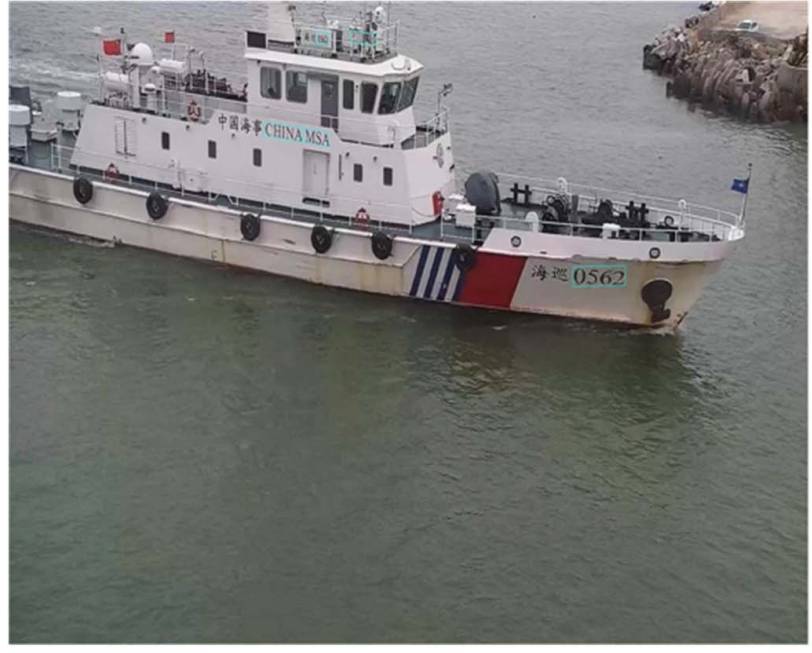

(A) EAST Result

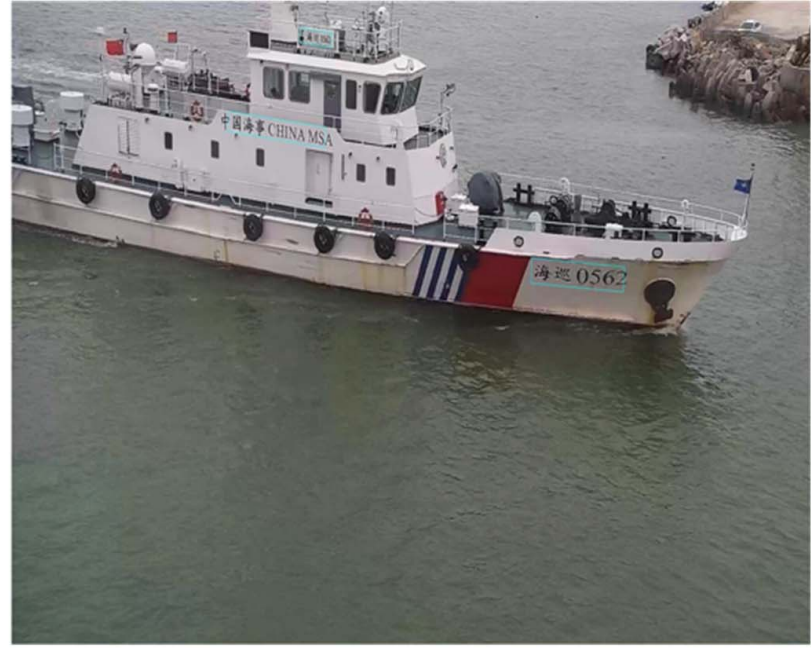

(B) FCNTLD Result

Figure 4: The test results of EAST and FCNTLD

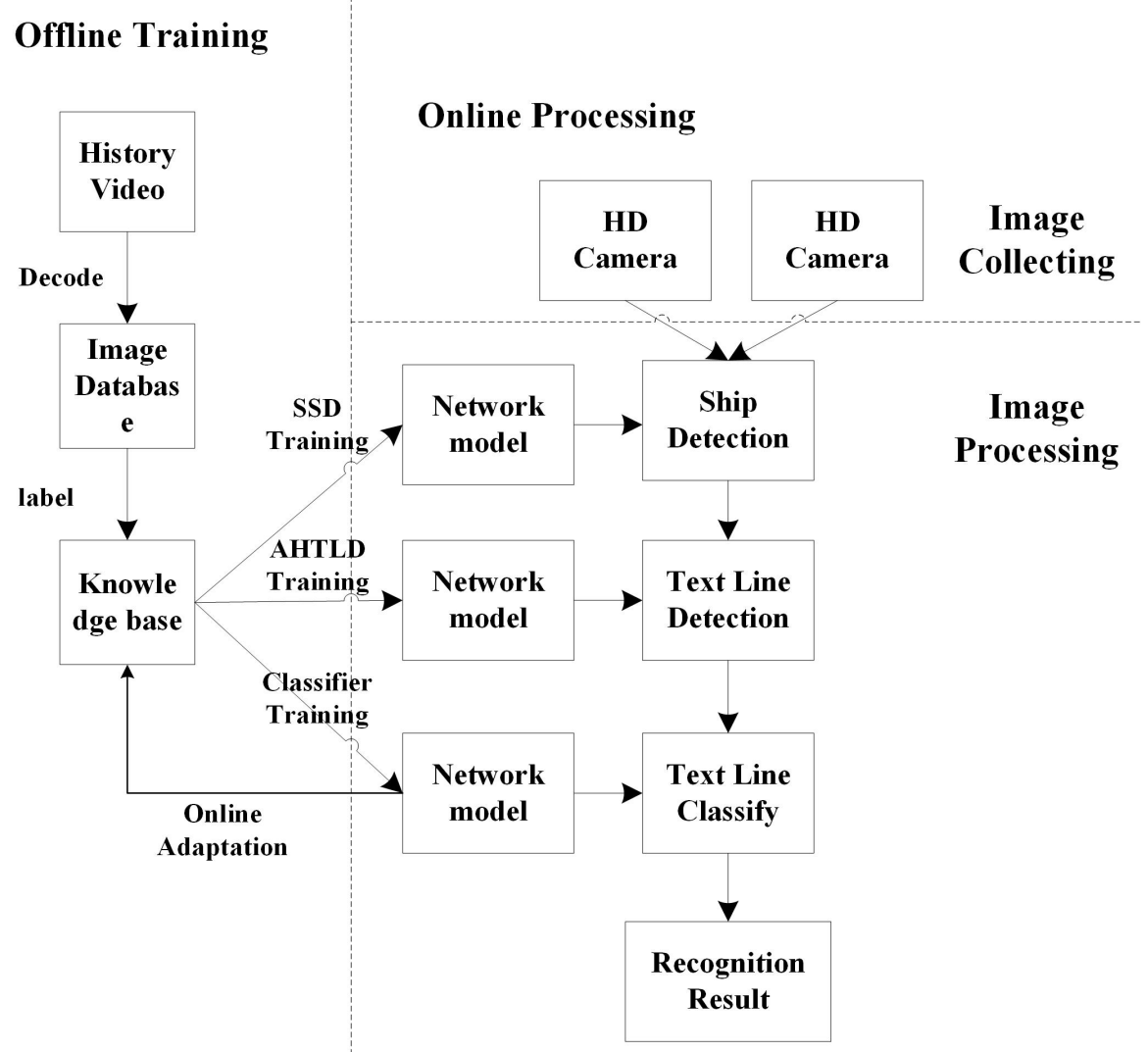

Figure 5: The architecture of implementing FCNPR 


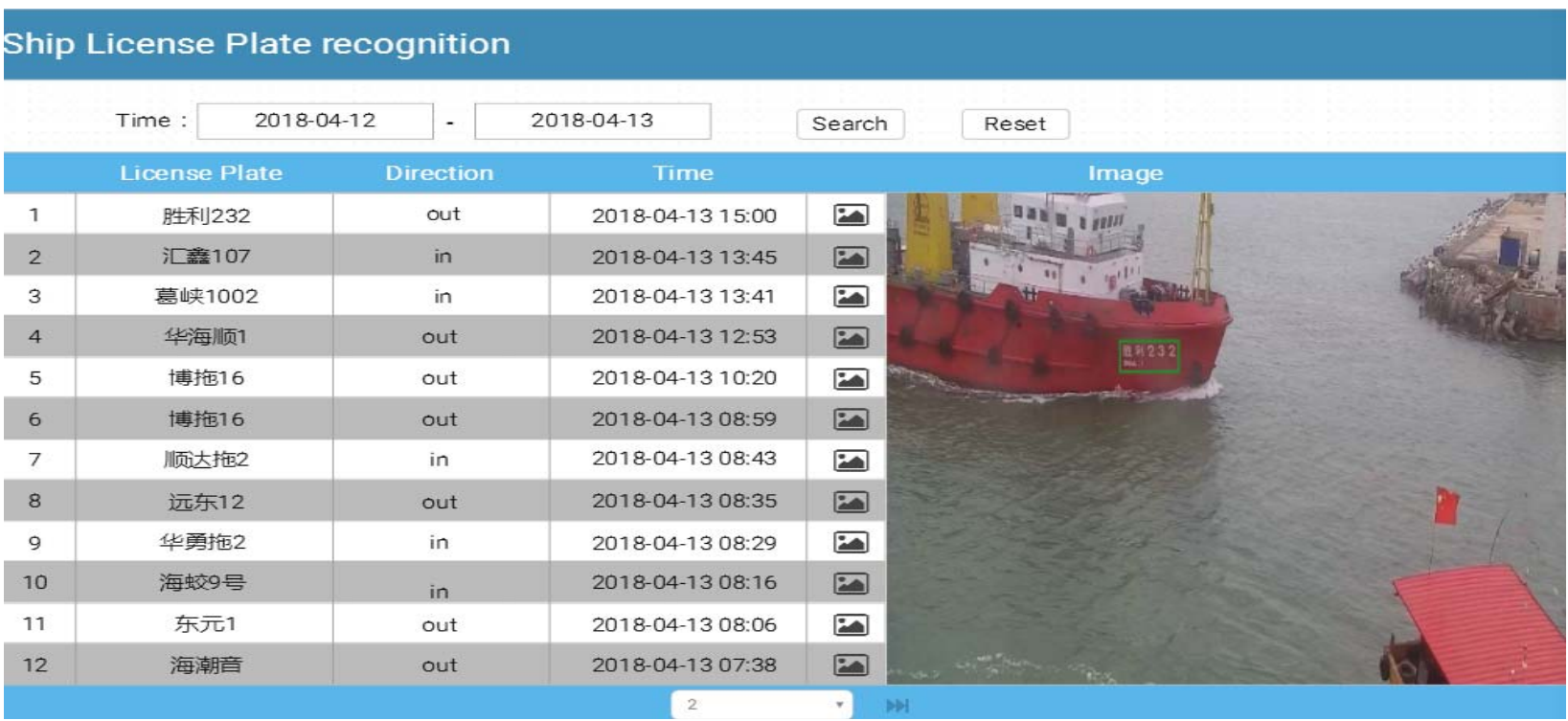

Figure 6: The FCNPR user interface

devices. The second layer detects ships, then, detects text lines, and recognizes ship plates.

\section{DePloyment AND RUNNING RESUlts}

The FCNPR is deployed in the port of Dongying, China. The video camera used at the scene is a Hikvision DS2CD3T25D-I5. The pixel is $1920 \times 1080$ and the frame rate is $30 \mathrm{fps}$. The camera is installed at the both sides of the entrance with the height of 8 meters. The server is a Nvidia $1080 \mathrm{Ti}$, desktop with 2 Duo $2.2 \mathrm{GHz}$ and 8GB RAM.

The results are shown in Fig. 6, including the recognized words, directions, time stamps, and on-site images.

\section{A. Performance}

We use the recall- $\mathrm{R}$ and precision-P as the evaluation criteria, which are defined as

$$
R=T P /(T P+F N), P=T P /(T P+F P)
$$

TP indicates true positive, $\mathrm{FN}$ indicates false negative, and FP indicates false positive. We collect 856 records within one month. We found there are 1048 ships passing through the port, and 148 ships have no license plate at all.

For ship detection, we collect 4700 images with ships in the SSD offline training phase. The performance of ship location with SSD is shown in Table I, where $S$ is the number of ships passing through the entrance, S-D indicates detect records, S-P indicates the positive detected S-D, S-T indicates the average time used for ship detection.

Table I: Accuracy of ship localization

\begin{tabular}{ccccccc}
\hline & S & S-D & S-P & P & R & S-T(ms) \\
\hline Ship Detection & 1048 & 1039 & 1031 & 0.9923 & 0.9838 & 44 \\
\hline
\end{tabular}

Table II shows the performance of text detection and plate recognition, which trained by AHTLD with 5900 images data. $\mathrm{T}$ is the total ships with license plate detected by SSD, T-D indicates the number of text detected from T, D-P indicates the positive result of T-D, D-T indicates the average time used for text detection.

Table II: Accuracy of text line detection

\begin{tabular}{ccccccc}
\hline & T & T-D & D-P & P & R & D-T(ms) \\
\hline Text Detection & 883 & 856 & 833 & 0.9731 & 0.9434 & 83 \\
\hline
\end{tabular}

Table III presents the recall and precision rates of text recognition. Specially, we collected 7300 images and divided into 103 categories for training the classifier. T-R indicates recognized text, R-P indicates the positive result of T-R, R-T indicates the average time of text recognition.

Table III: Accuracy of text line recognition

\begin{tabular}{ccccccc}
\hline & D-P & T-R & R-P & P & R & R-T(ms) \\
\hline Text Recognition & 883 & 826 & 794 & 0.9613 & 0.9532 & 24 \\
\hline
\end{tabular}

We summarize the total recall and precision rates, and total time (T-T) every 7 days in Table IV. The recall and precision rates increase as time goes on, which proves the Online Adaptation is effective.

\section{Conclusions}

This paper proposes a FCN based approach for ship plate recognition (FCNPR). It first locates ships using the SSD, and then detects plate text lines with FCNTLD, and adopts the text line classifier to recognize plate text. FCNPR also integrates 
Table IV: Records of adaptability

\begin{tabular}{cccc}
\hline days & $\mathrm{P}$ & $\mathrm{R}$ & $\mathrm{T}-\mathrm{T}(\mathrm{ms})$ \\
\hline 7 & 0.8592 & 0.8233 & 156 \\
14 & 0.8932 & 0.8651 & 156 \\
21 & 0.9145 & 0.8864 & 157 \\
28 & 0.9278 & 0.8922 & 156 \\
\hline
\end{tabular}

the AIS information to enrich the knowledge base for updating the text line classifier online. We implement the FCNPR with an adaptive architecture supporting real-time video processing, and deploy the system at the Dongying Port, China. The running results in the past 6 months show its effectiveness.

In the future, we will adopt a multi-model data fusion approach [13] [14] to improve plate recognition accuracy.

\section{ACKNOWLEDGMENT}

This work was supported in part by the Key Research Program of Shandong Province under Grant 2017GGX10140 and in part by the Fundamental Research Funds for the Central Universities(15CX08015A), National Natural Science Foundation of China (No. 61309024).

\section{REFERENCES}

[1] P. M. Alderton, "Port management and operations," Harbors, 2008.

[2] L. Xu, J. S. J. Ren, C. Liu, and J. Jia, "Deep convolutional neural network for image deconvolution," in International Conference on Neural Information Processing Systems, 2014, pp. 1790-1798.

[3] J. Long, E. Shelhamer, and T. Darrell, "Fully convolutional networks for semantic segmentation," in IEEE Conference on Computer Vision and Pattern Recognition, 2015, pp. 3431-3440.

[4] A. Haratimokhtari, A. Wall, P. Brooks, and J. Wang, "Automatic identification system (ais): Data reliability and human error implications," Journal of Navigation, vol. 60, no. 3, pp. 373-389, 2007.

[5] C. Gou, K. Wang, Y. Yao, and Z. Li, "Vehicle license plate recognition based on extremal regions and restricted boltzmann machines," IEEE Transactions on Intelligent Transportation Systems, vol. 17, no. 4, pp. 1096-1107, 2016.

[6] G. U. Hui and Y. Wang, "Study on method of distortion correction in ship license plate recognition system," Modern Electronics Technique, 2009.

[7] D. Lin, F. Lin, Y. Lv, F. Cai, and D. Cao, "Chinese character captcha recognition and performance estimation via deep neural network," $\mathrm{Neu}$ rocomputing, 2018.

[8] R. Smith, "An overview of the tesseract ocr engine," in International Conference on Document Analysis and Recognition, 2007, pp. 629-633.

[9] X. Zhou, C. Yao, H. Wen, Y. Wang, S. Zhou, W. He, and J. Liang, "East: An efficient and accurate scene text detector," in IEEE Conference on Computer Vision and Pattern Recognition, 2017, pp. 2642-2651.

[10] S. Wang, J. Sun, and S. Naoi, "On study of the reliable fully convolutional networks with tree arranged outputs (TAO-FCN) for handwritten string recognition," CoRR, vol. abs/1707.02975, 2017. [Online]. Available: http://arxiv.org/abs/1707.02975

[11] W. Liu, D. Anguelov, D. Erhan, C. Szegedy, S. Reed, C. Y. Fu, and A. C. Berg, "Ssd: Single shot multibox detector," in European Conference on Computer Vision, 2016, pp. 21-37.

[12] DING and Xiao-qing, "Chinese character recognition: A review," Acta Electronica Sinica, 2002.

[13] W. Zhang, Y. Zhang, J. Zhai, D. Zhao, L. Xu, J. Zhou, Z. Li, and S. Yang, "Multi-source data fusion using deep learning for smart refrigerators," Computers in Industry, vol. 95, pp. 15-21, 2018. [Online]. Available: https://doi.org/10.1016/j.compind.2017.09.001
[14] W. Zhang, Z. Wang, X. Liu, W. Gong, H. Sun, J. Zhou, and Y. Liu, "Deep learning based real-time fine-grained pedestrian recognition using stream processing," IET Intelligent Transport Systems, 2018. 